\title{
The Impact of Mobile Application Support on the Quality of Care of Kidney Patients
}

\author{
Arwa A. Jamjoom ${ }^{1}$ \\ ${ }^{1}$ King Abdulaziz University, Jeddah, Saudi Arabia \\ Correspondence: Arwa A. Jamjoom, King Abdulaziz University, Jeddah, Saudi Arabia. E-mail: \\ ajamjoom@kau.edu.sa
}

Received: April 10, 2018

Accepted: April 16, $2018 \quad$ Online Published: April 30, 2018

doi:10.5539/cis.v11n2p99

URL: https://doi.org/10.5539/cis.v11n2p99

\begin{abstract}
The adaptation of mobile application is supportive for the discharge patients in less amount of time. The use of mobile application enables physicians to detect the possible implications of treatment at an early stage. The study aims to provide a support and prevention application for kidney failure patients to monitor their diet, receive notifications for taking medicines, get a reminder of dialysis, and conduct easy communication between the doctors and patients. For this purpose, the study has supported the implementation and usage of Kidney Failure Care Application (KFCA). The software architecture of this application aids in the understanding of how the system would behave. The results have shown that the application has eased the communication with their respective doctors, and their diet was also monitored effectively. An average success rate of $80 \%$ was observed as a result of testing KFCA application.
\end{abstract}

Keywords: mobile application, kidney patients, glomerular filtration rate (GFR), kidney failure care application (KFCA)

\section{Introduction}

The progression of kidney disease is slow and can be further slowed, if diagnosed at an early stage. The kidney function is accurately evaluated by measuring Glomerular Filtration Rate (GFR). GFR is calculated by a formula using an individual's age, race, gender, and serum creatinine (Schwartz et al., 2009). Creatinine is excreted as a waste product through muscle activity. Kidneys are responsible for excreting creatinine from the body; however, if the kidney function is impaired, the level of creatinine increases in the bloodstream (Cooper, 2016). The standard functionality of any kidney disease management tool helps the affected patients to live normal life.

Majority of the kidney failure patients are diagnosed after they reach the later stages; i.e. stage 3 and 4. Moreover, majority of the patients face problems regarding their diet and are not aware about what should they eat to avoid medical complications. At times, patients need advice about the use of drugs, but they are unable to get proper information due to poor communication with the doctor. Becker, et al. (2013) examined the impact of mobile applications on enhancing the efficiency of healthcare quality care services. The subjects include individuals receiving drug therapy services. Drug intake and level of chronic disease were monitored and examined through an iOS app. The patients appreciated the adaptation of iOS app for monitoring the levels of drug intake. As per the findings, the use of mobile application has improved the quality care services.

The previous applications; including TRKRR and My Food Coach application, have resulted in significant improvement in the diet of patients. However, another application 'H2OVERLOAD' has been designed to limit the fluid intake among patients with hyponatremia. It created alert signals for the patients, if weight or blood pressure increases above the normal level. Therefore, the present study aims to provide a support and prevention application for the kidney failure patients to monitor their diet and conduct easy communication between the doctor and patients. The study is significant as it would help these patients to minimize medical complications through proper awareness and monitor their diet and fluid intake. The system would effectively monitor the intake of sodium, potassium, and phosphor to help the kidney disease patients improve their life routine.

\section{Literature Review}

A study has examined the role of mobile applications in improving the recovery and quality care of patients. The findings revealed that the use of mobile applications in healthcare sector can improve the quality care procedures. 
Moreover, it is also suggested that the use of mobile applications can be highly feasible in healthcare sector. The recipients of the technology are showing high acceptance level and recommending the future use of mobile applications support (Semple, et al. 2015). Similarly, Browning, et al. (2016) conducted a research to examine the role of mobile technology in healthcare sector. The study stated that the use of mobile health technology and applications is significant in re-shaping the healthcare industry. Mobile applications are proving to be helpful in monitoring the kidney symptoms among patients at an early stage. A survey was conducted on the patients receiving treatment for kidney transplant by Skelton et al. (2015). A total of 139 patients participated in the survey. As per the results, the patients showed a positive attitude and behavior towards mobile healthcare applications (Skelton et al., 2015).

Israni et al. (2016) have investigated whether the use of mobile application enhances the patient adherence rate or not. The qualitative research technique was adopted, and the patients receiving kidney transplant services were included. As per the findings, the recipients of kidney transplant understood the significance of clinical medications for improving the chronic disease. Subjects showed significant acceptance level in the adaptation of mobile application support in healthcare sector. The study suggested that potential barriers in the adaptation of mobile applications needed to be improved. Furthermore, hospital management or medical staff should provide guidance to the patients in using mobile applications. Jamison et al. (2016) examined the impact of mobile applications in improving the quality care services. Mobile application was developed to identify and monitor the chronic pains of the patients. The mobile application was developed for iPhone and Android users. A total of 90 patients, suffering from chronic pain; males and females, were included in the study. The findings revealed that the use of mobile applications did not contribute towards reducing the level of pain among patients. The patients, who were satisfied with the mobile application, indicated that their pain levels have increased. However, it was observed that the use of mobile had brought a positive change in the moods and behaviors of the patients.

Jamison et al. (2017) analyzed the impact of mobile phone application to monitor pain. The study has focused on assessing the performance of mobile application and its effectiveness in communicating with the recipients of the device. The participants included 105 patients, suffering from chronic pain. The overall progress of every patient was recorded for continuous three months. As per the findings, the pain rate was calculated as 0.69 , sleep rate as 0.83 , and reliability and validity of standardized questionnaires was recorded as 0.50 . The recipients of the application showed higher level of satisfaction. The patients of chronic pain were able to easily communicate their pain level to the medical doctors and physicians. Armstrong et al. (2014) has evaluated the role of mobile application support in improving quality care. The study focused on examining the contribution of mobile homebased apps in reducing the follow up visits of patients. The findings suggested that the adaptation of mobile application is cost effective. The mobile application proved to be effective in reducing the follow-up visits. The patients were able to communicate with their physicians from home, which decreased the need for visiting physicians to a significant level. Patients using the mobile application showed positive behavior and high satisfaction level.

\section{Methodology}

Agile method has been used to develop solutions that mainly focus to deliver high-quality working software. It tends to increase business value and minimizes the project overhead. It welcomes any changing requirements in its development for achieving maximum competitive advantage. The phases of development of agile cycle has been illustrated in Figure 1. The planning phase involves the breakdown of idea into smaller pieces of work; while, the requirement analysis is concerned with gathering information regarding the product usage. The preparation of software design depends on it that can result in the development of test strategy. The implementation phase is all about the creation and testing of certain features. The product is delivered to the customers after going through the testing phase. 


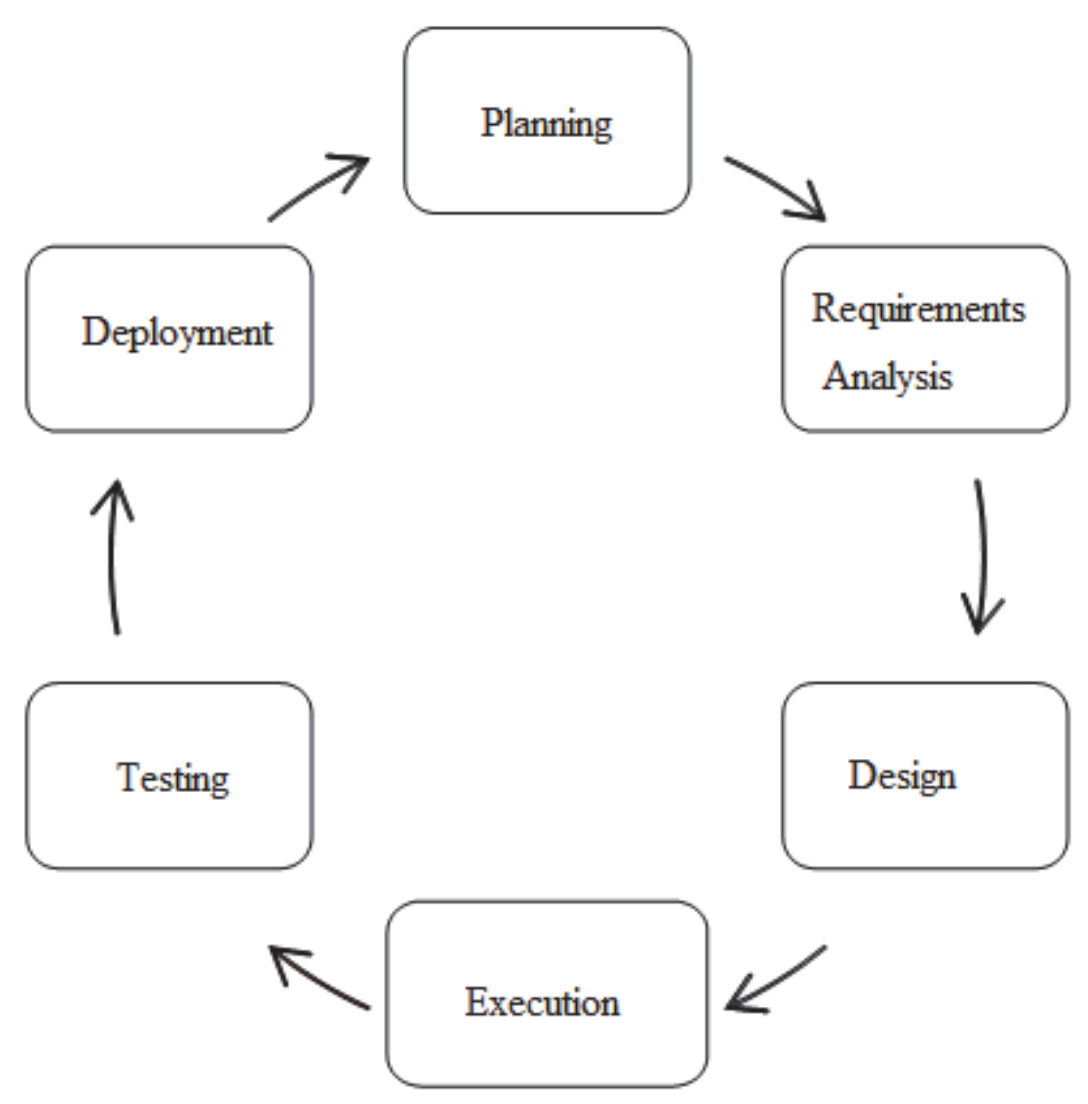

Figure 1. Development of Agile

\section{Analysis}

The data was collected through online questionnaire that showed that majority of the doctors have faced problems as the patients are not committed to their diets, medicines, fluid control, and dialysis time. Therefore, the patients need reminder for their medicines and appointment time, aware about the periodic lab results, and manage their diet chart. An interview was conducted to understand the process of dialysis and gather clear requirements from the user of Kidney Failure Care Application (KFCA). Figure 2 has illustrated the overview of functional requirements and factors of KFCA. 


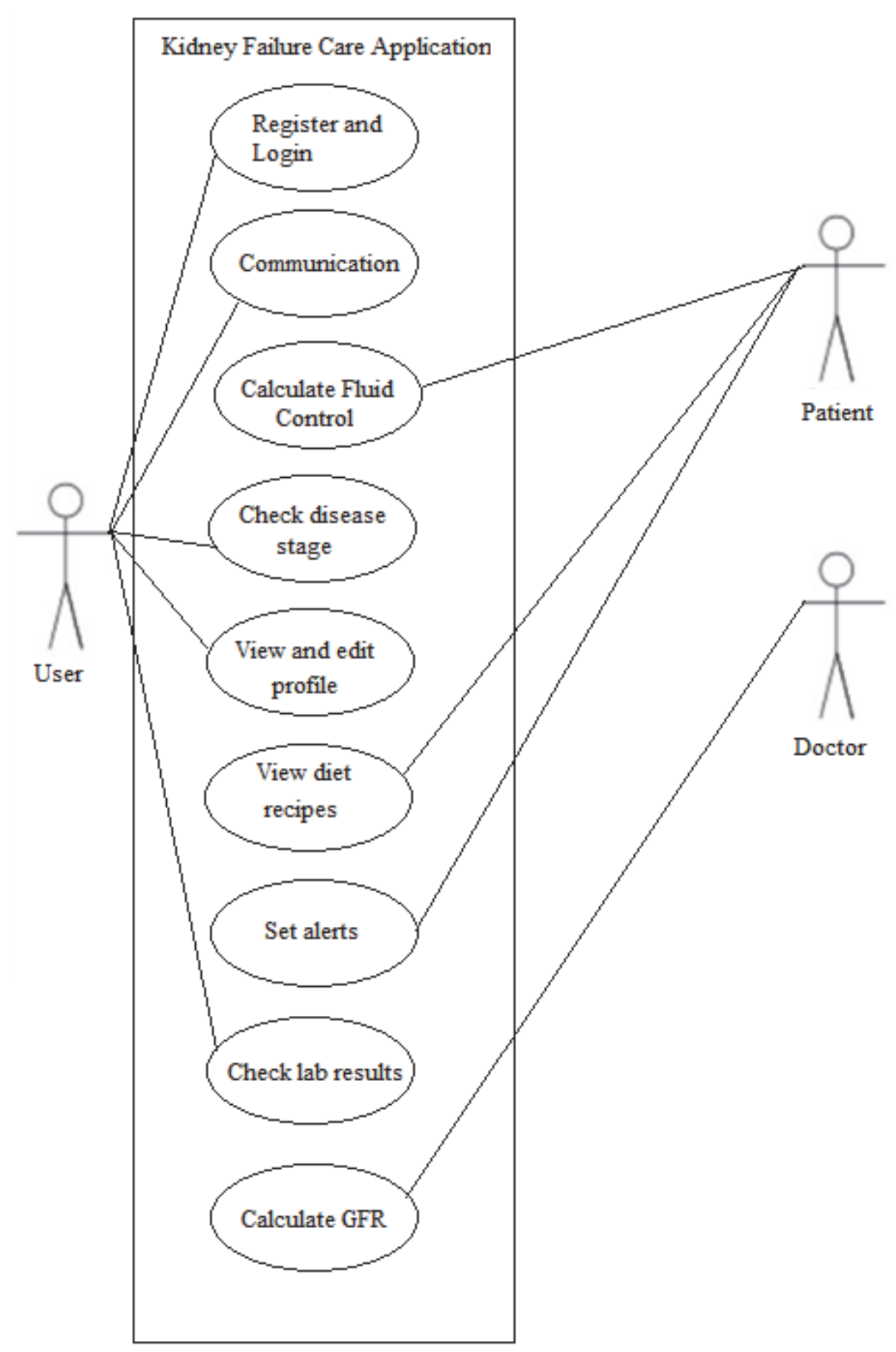

Figure 2. Functionality of KFCA

The application starts as the user registers and fills his/her information; and the application also verifies and checks the information. Afterwards, the user can create account and can login by entering the username and password. The patient can select alert for his/her medicines and set the time, which will be intimated when the medication scheduled. This sequence has the similar function of setting for the dialysis time. The patient can calculate his/her fluid control through selecting 'calculate fluid control' and enter the amount of fluid excreted out from the body (urine). The communication between doctor and patient can be carried through online chat. The user will select the contact whether a doctor or patient, then write a message and send it. Moreover, the user can check the lab results through 'lab results function'. 


\section{Architecture and Design of Kidney Failure Care Application (KFCA) System}

The software architecture of a computer system is fundamentally the depiction of system that aids to understand how the system will behave. Figure 3 has shown entire functioning of application and its connection to the database by electronical channel system. Service Realization viewpoint has shown how the underlying processes are identified by one or more business services. It builds up an association between business process view and business products viewpoint.

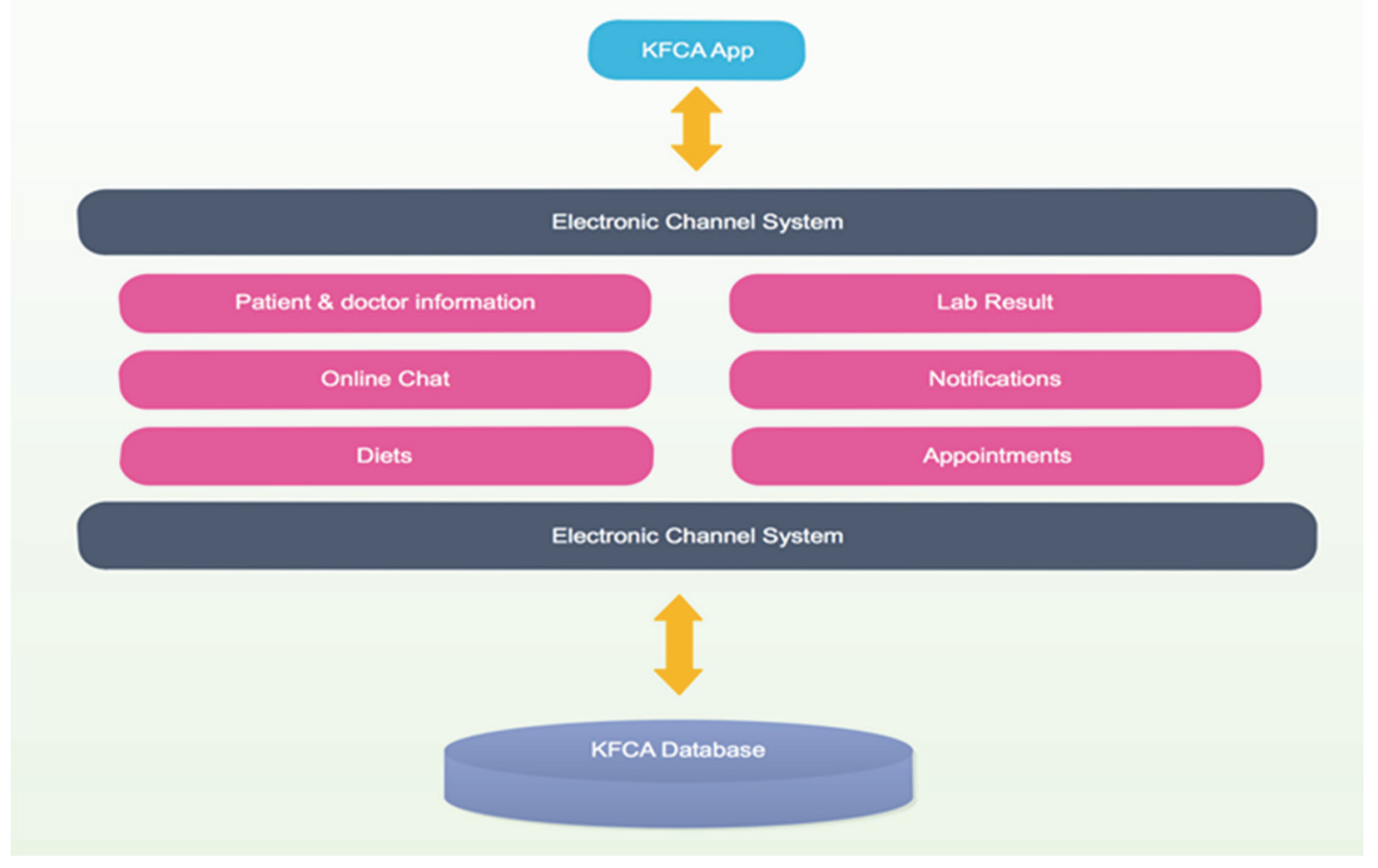

Figure 3. Architecture of KFCA Application

High level of structures and composition of one or more business processes have been shown by the business process. Dataflow is termed as the movement of data through a system that comprise of software, hardware, or a combination of both. The entire process of data passes from one component to the next within a program or a system. Table 1 has shown the heuristics to design the user interfaces.

Table 1. Ten Heuristics to design user interfaces by Jakob Nielsen

\begin{tabular}{lll}
\hline Heuristics for Designing & Description Feature & Description \\
User interfaces & & \\
\hline
\end{tabular}

1. Visibility of system The system is responsible status

2. Match between system and the real world for informing the users about what is going on via suitable feedback in a specified time limit.

The language of user needs to be spoken by the system in words and phrases. This concept is acquainted with the user, as compared to the system-oriented terms. The information is appeared in natural and logical order by
This Feature is Applied

This Feature is Applied

The application interface has language (Arabic) and information present in coherent, consistent form. 


\begin{abstract}
following the real-world conventions.

3. User control and Users need 'emergency Freedom exit', when they choose system functions by mistake. They want to leave that unwanted selection without going through a long procedure.

4. Consistency and The users need not to standards worry about different words or phrases that mean the same.
\end{abstract}

\section{Error prevention}

6. Recognition rather than recall

Flexibility

efficiency of use

8. Aesthetic minimalist design
The error messages provide an appropriate design to prevent the occurrence of any problem at first place. The users are presented with confirmation option before committing to an action by eliminating error-prone condition or checking them properly.

The visibility of objects and actions minimizes user's memory load. It helps the user as the user need not to memorize information from one part of the dialogue to another. Instructions regarding the use of system need to retrieved easily, whenever needed.

and The visibility of objects and actions minimizes user's memory load. The information from one part of the dialogue to another is not memorized by the user. Moreover, instructions are needed for using the system appropriately.

nd Any unwanted and irrelevant information is not included in the dialogues. It is because the irrelevant information within a dialogue competes with the relevant knowledge, which diminish their
This Feature is Applied

This Feature is Applied

The default is on Home and the symbols is clear for each subject.

This Feature is Applied

When the user enters wrong values (e.g. Add food) the system will alert the user to allow user to resume it.

\section{This Feature is Applied}

Reduce required actions and sequences because the user need in recalling or remembering the users' guidelines and provide help options in the games.

This Feature is Applied.

For both patient and doctors know literate

This feature is applied

Easy, related, well- organized visuals and few labels would create active interaction between the interface and customers. 
relative visibility.

9. Help users recognize, The messages showing diagnose, and recover error need to be expressed from errors

10. Provision of help and documentation in plain language without any codes. It helps in indicating the problems precisely and also suggests an appropriate solution for it.

It may be necessary to provide help and documentation; although, it is better if the system can be used without documentation. This type of information should be easy to search, by focusing on user's task.
This feature is applied

When the user enters incorrect number, it will not show the user where is the mistake

This feature is not

applied in all application because we don't have search function in application

\section{Implementation and Testing of KFCA System}

Android studio was used as a software in KFCA application as its provides a useful tool like drag and drop. It is flexible and easy to connect to the database. MySQL was used to save money and time to power their high-volume. Laptop was used to download android studio software to develop KFCA application and smartphone was used to test its functions. The configuration of run time processing nodes and the components that live on them are depicted through a UML deployment diagram. The diagram is used to present the physical aspects of an object-oriented system. Figure 4 has shown the connection of different components with each other.

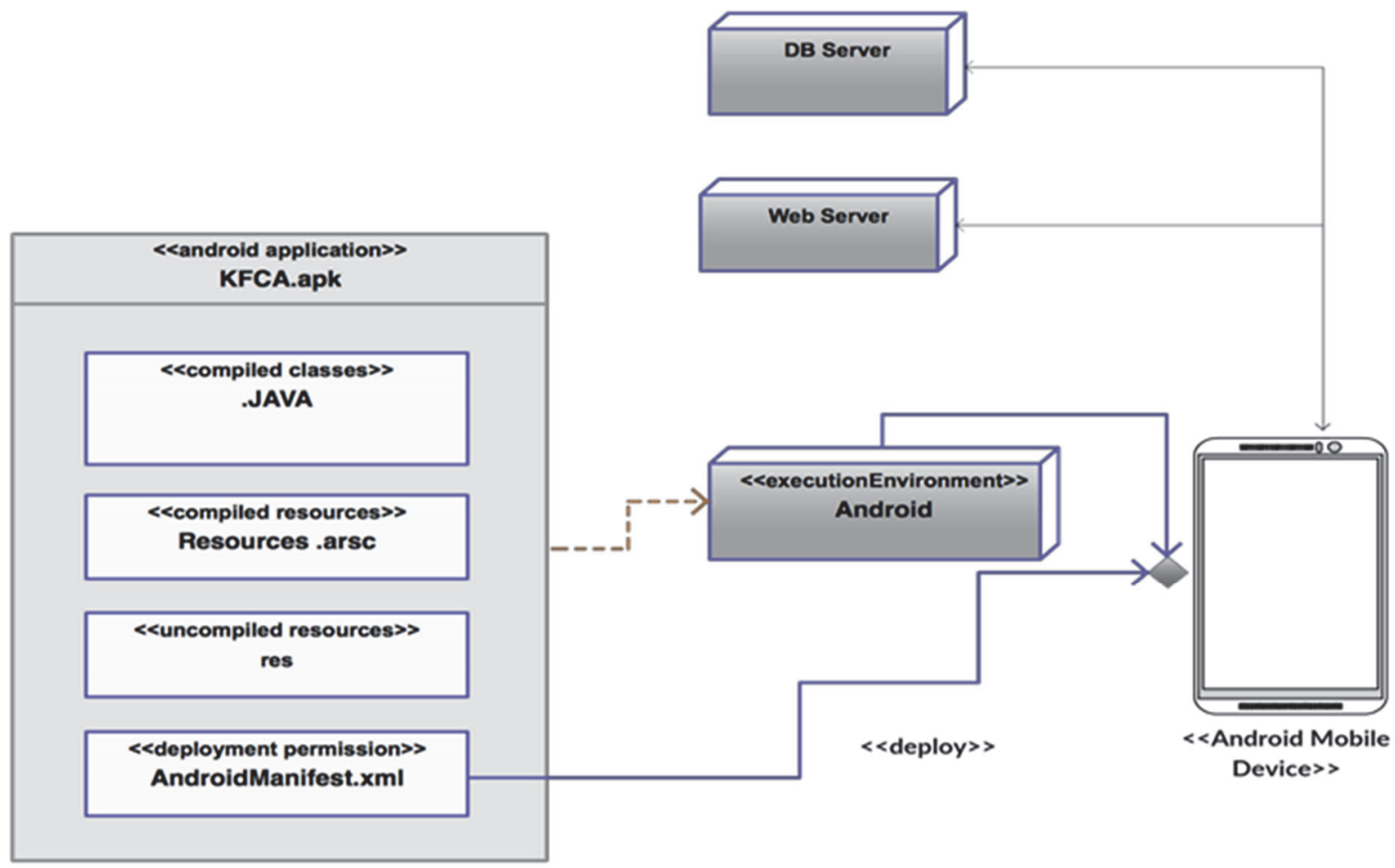

Figure 4. Deployment of KFCA application 


\section{Discussion}

The results have shown that application of KFCA successfully meet the user's requirements. The patients were successfully applied to communicate with their respective doctors and their diet was also monitored effectively. The interfaces, used in KFCA, are user friendly and suitable for the patients as well as the doctors. An average success rate of $80 \%$ was observed as a result of testing KFCA application.

The use of technology in medical and healthcare practices has evolved with the passage of time. The mobile application is a reliable and efficient tool providing better customer care to the patients. The use of technology has contributed towards the enhancement of cooperation and collaboration level between the patients and hospital administration. It also provides relevant support to the patients. According to Boulos, et al. (2014); the use of mobile applications in hospitals assists in improving the quality care services. Martinez-Perez et al. (2014) examined the impact of mHealth and mobile application support in improving the healthcare services. The mobile application support has facilitated in the clinical and customer care processes. The research has focused on examining the previous studies, conducted in this domain. Numerous apps on AppStore and Google Play related to healthcare and medical services were examined. The patients showed a positive attitude towards mobile application support. The patients showed higher acceptance level to the adaptation of mobile technology in healthcare sector. The technology helped the kidney patients in making informed decisions about the clinical processes.

McGillicuddy et al. (2013) has analyzed the behaviors of kidney transplant patients towards the mobile technology. The purpose was to assess the efficiency of mobile application support in healthcare and examine the acceptance level of the kidney transplant recipients. As per the results, the recipients of the mobile technology showed positive attitude towards the technology. The use of mobile technology in healthcare enables the kidney transplant patients to make better clinical decisions. Moreover, majority of the renal transplant patients recommended that the mobile based technology should be incorporated in healthcare center.

Diamantidis and Becker (2014) have examined the role of mobile technology in improving the communication and engagement levels patients receiving treatment for kidney disease. The mobile applications facilitated the patients in their treatment, as the patients were able to monitor their dietary routine and intake, analyze the kidney functioning, and communicate effectively with healthcare personnel. The mobile health and health information mobile applications provided relevant information to the patients receiving treatment for chronic kidney illness. The adaptation of mobile application support enhanced the quality care services. The mobile technology provides reliable solutions to the kidney patients. Deglise et al. (2012) analyzed the effectiveness of using mobile technology in healthcare sector by focusing on SMS based applications for better management and monitoring of healthcare services. Numerous healthcare applications related to disease surveillance, prevention, compliance, and management applications were examined. The findings revealed that there is a higher level of acceptance in the adaptation of mobile technology. The mobile application support provided an efficient communication method to the patients receiving treatment for kidney disease.

Singh et al. (2016) examined the role of mobile applications in healthcare industry for improving the engagement level of the patients. Multiple Android and iOS apps were analyzed, and the findings revealed that the adaptation of mobile applications in healthcare produce high level of patient engagement. The consumers found the applications to be useful in monitoring various components of their chronic kidney illness. Furthermore, the mobile applications resulted in improving the efficiency of quality care services, as the patients were able to collaborate with the physicians. Ong et al. (2015) investigated the use of technology in healthcare sector results to improve the quality care services. The adaptation of mobile applications and latest technology in hospitals enabled the authorities to effectively manage quality care procedures. The results suggested that the recipients of technology appreciated the use of mobile technology in healthcare sector. The mobile technology-based management tools result in providing quality healthcare services to the patients, suffering from renal diseases. Future work needs to focus on adding google map in appointment notification to facilitate patients, in reaching the place. The application needs to notify the patients, when he/she exceeds the allowed number of certain elements.

\section{Acknowledgments}

The author is thankful to all the associated personnel, who contributed in/for the purpose of this research.

\section{References}

Armstrong, K. A., Semple, J. L., \& Coyte, P. C. (2014). Replacing ambulatory surgical follow-up visits with mobile app home monitoring: modeling cost-effective scenarios. Journal of medical Internet research, 16(9). 
https://doi.org/10.2196/jmir.3528

Becker, S., Kribben, A., Meister, S., Diamantidis, C. J., Unger, N., \& Mitchell, A. (2013). User profiles of a smartphone application to support drug adherence-experiences from the iNephro project. PloS one, $8(10)$, e78547. https://doi.org/10.1371/journal.pone.0078547

Browning, R. B., McGillicuddy, J. W., Treiber, F. A., \& Taber, D. J. (2016). Kidney transplant recipients' attitudes about using mobile health technology for managing and monitoring medication therapy. Journal of the American Pharmacists Association, 56(4), 450-454. https://doi.org/10.1016/j.japh.2016.03.017

Cooper, D. (2016). Monitoring for renal dysfunction. Critical Care Nursing: Monitoring and Treatment for Advanced Nursing Practice, 104-118. https://doi.org/10.1002/9781118992845.ch7

Israni, A., Dean, C., Kasel, B., Berndt, L., Wildebush, W., \& Wang, C. J. (2016). Why do patients forget to take immunosuppression medications and miss appointments: can a mobile phone app help? JMIR public health and surveillance, 2(1). https://doi.org/10.2196/publichealth.5285

Jamison, R. N., Jurcik, D. C., Edwards, R. R., Huang, C. C., \& Ross, E. L. (2017). A pilot comparison of a smartphone app with or without 2-way messaging among chronic pain patients: who benefits from a pain app? The Clinical journal of pain, 33(8), 676. https://doi.org/10.1097/ajp.0000000000000455

Jamison, R. N., Mei, A., \& Ross, E. L. (2016). Longitudinal trial of a smartphone pain application for chronic pain patients: Predictors of compliance and satisfaction. Journal of telemedicine and telecare, 1357633X16679049. https://doi.org/10.1177/1357633x16679049

Schwartz, G. J., Munoz, A., Schneider, M. F., Mak, R. H., Kaskel, F., Warady, B. A., \& Furth, S. L. (2009). New equations to estimate GFR in children with CKD. Journal of the American Society of Nephrology, 20(3), 629637. https://doi.org/10.1681/asn.2008030287

Semple, J. L., Sharpe, S., Murnaghan, M. L., Theodoropoulos, J., \& Metcalfe, K. A. (2015). Using a mobile app for monitoring post-operative quality of recovery of patients at home: a feasibility study. JMIR mHealth and uHealth, 3(1). https://doi.org/10.2196/mhealth.3929

Skelton, S. L., Waterman, A. D., Davis, L. A., Peipert, J. D., \& Fish, A. F. (2015). Applying best practices to designing patient education for patients with end-stage renal disease pursuing kidney transplant. Progress in Transplantation, 25(1), 77-90. https://doi.org/10.7182/pit2015415

\section{Copyrights}

Copyright for this article is retained by the author(s), with first publication rights granted to the journal.

This is an open-access article distributed under the terms and conditions of the Creative Commons Attribution license (http://creativecommons.org/licenses/by/4.0/). 